\title{
GESTURE-BASED SMART CAR CONTROL SYSTEM
}

\author{
Yang-Keun Ahn, and Young-Choong Park \\ Korea Electronics Technology Institute \\ 121-835, 8th Floor, \#1599, Sangam-Dong, Mapo-Gu, Seoul \\ Republic of Korea
}

\begin{abstract}
This article describes a method of controlling a screen showing output information on a transparent display of a car windshield by using a single depth camera without substantial touches made by user. First, this article introduces a smart car menu control system that may be used to control a smart automobile through spatial gesture interaction. Then, the method of extracting user's hand region and recognizing gestures is described. Gesture recognition rate was measured experimentally to verify the reliability of the system proposed in the present study.
\end{abstract}

\section{KEYWORDS}

Hand Detection, Fingertip Detection, Gesture Recognition, Air Touch, Screen Control System

\section{INTRODUCTION}

As various electronic devices have been developed, smart devices may be easily found in all areas of daily living. A variety of smart devices, including smart phones, smart watches, smart TVs, and even smart cars, have appeared. One representative example of smart car services is Advanced Driver Assistance Systems (ADAS), which allows autonomous driving.

Not only electronic devices but also display technologies have been developed. While conventional LCD monitors were just for viewing information shown on a screen, transparent displays that are currently under development enable driver to view what is behind the screen .

This article proposes a method of replacing the windshield glass of a car with a transparent display and controlling output information shown on the front screen. Direct touching of the information displayed on the windshield while driving may cause safety problems. Therefore, this article proposes a method of controlling the screen with only user gestures, without directly touching the front display.

Figure 1 shows the smart car system that enables user to control the menu through spatial interaction during driving. Figure 2 is the overall system flowchart showing the process of spatial interaction.

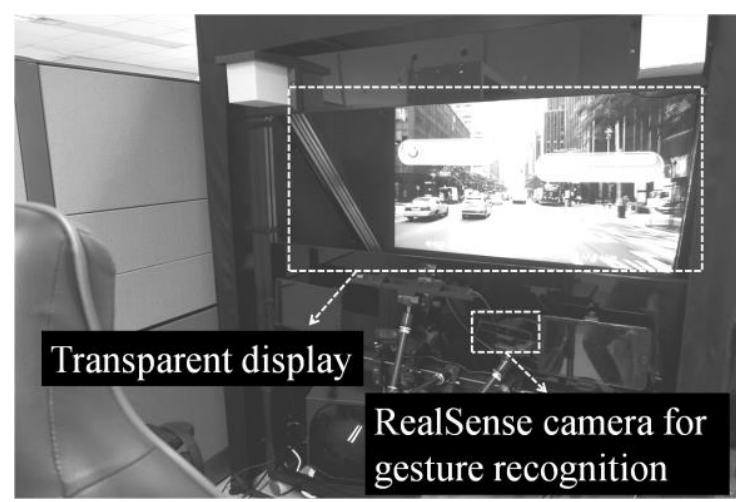

Figure 1. System architecture 


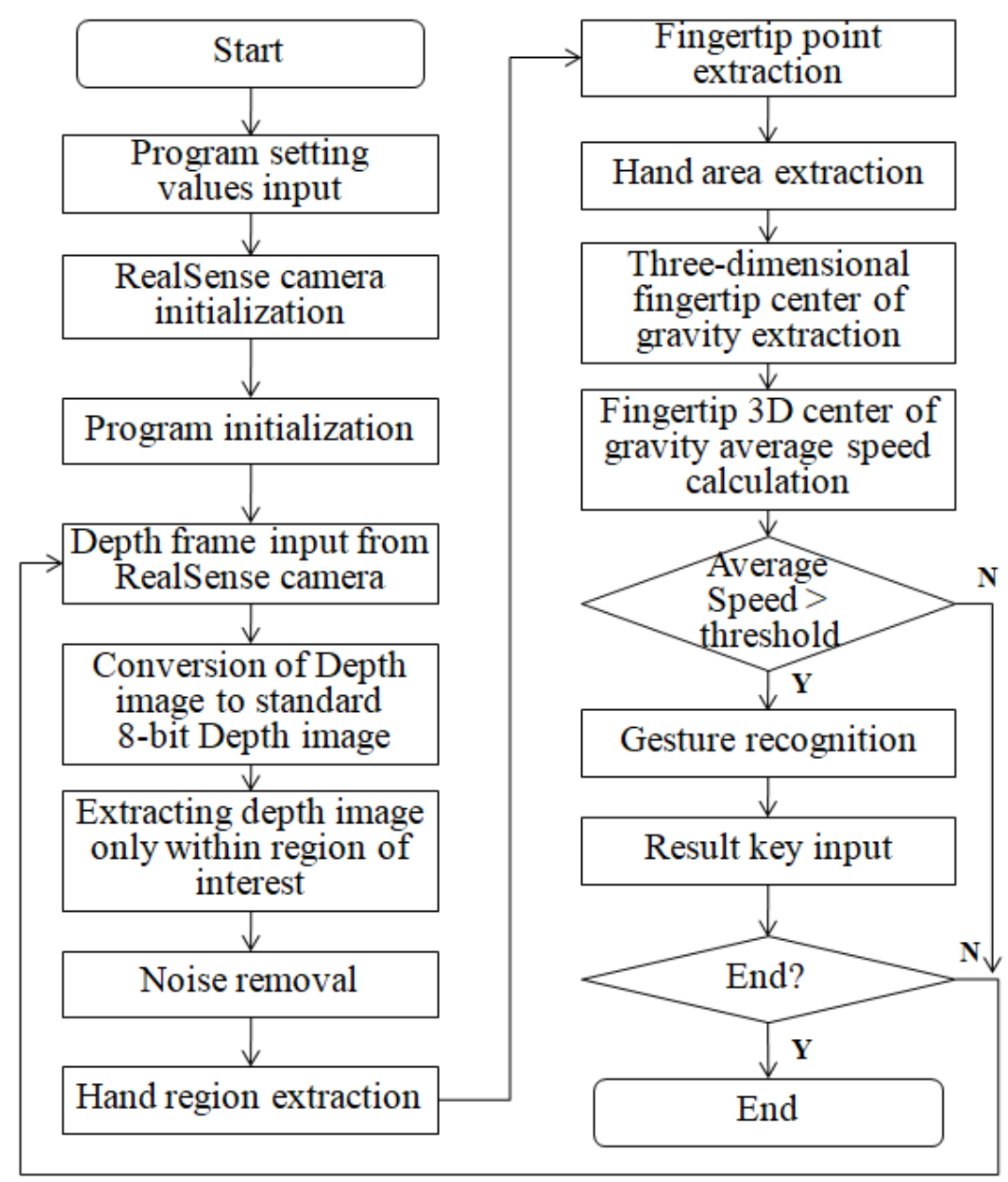

Figure 2. Overall System flowchart

\section{REFERENCE POINT EXTRACTION}

Before recognizing a user's gesture, a reference point for recognizing gesture speed should be extracted. In the present study, the reference point for measuring hand speed was defined as the center of gravity of the user's hand region.

To extract the hand region, the depth values within the limits of a depth camera were binarized. Widening the allowance limits of the depth camera may cause the image to include a user's elbow region, which causes an erroneous recognition of the elbow as the center of gravity of the hand while the hand region is moving. In the present study, the hand region extraction range was limited to a region within a $40 \mathrm{~cm}$ radius with reference to the front center of the depth camera.

A smoothing calculation should be performed to prevent erroneous operation due to motion blur after the extraction of the hand region. In the present study, a dilation calculation was performed eight times to remove noise, and the results showed that almost no erroneous operation was caused by motion blur even in rapid gesture actions. 


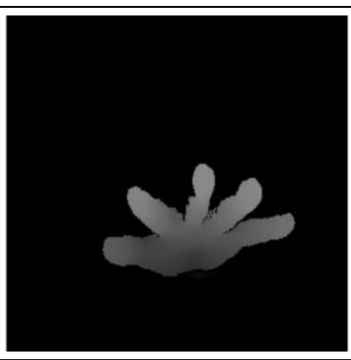

(a)

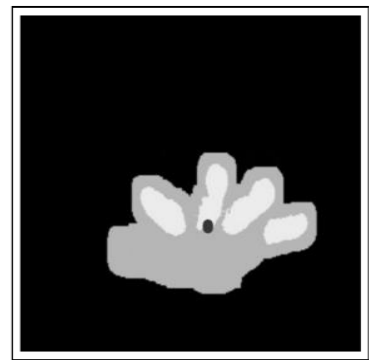

(b)

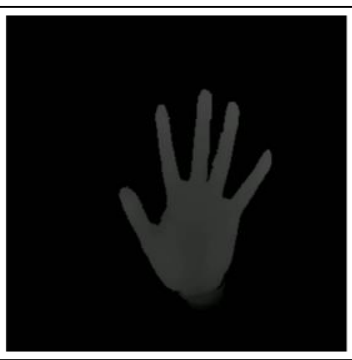

(c)

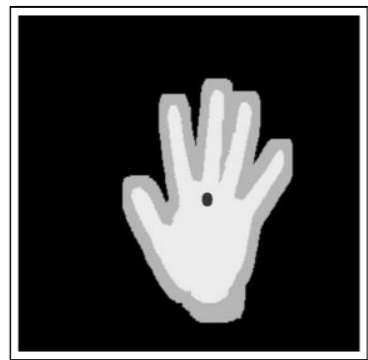

(d)

Figure 3. Extraction of reference point and area

(a) A depth image; (b) reference point and area information of depth image(a);

(c) A depth image; (d) reference point and area information of depth image(c)

The information used for gesture recognition includes the three-dimensional $\mathrm{X}, \mathrm{Y}$, and $\mathrm{Z}$ coordinate values and the area of the fingertip region. The $\mathrm{X}$ and $\mathrm{Y}$ coordinate values of the hand region may be obtained by using the secondary moment. The $\mathrm{Z}$ coordinate value was obtained by averaging the depth values of the entire extracted hand region. The reference point of the hand region is the gray point shown in Figures 3(b) and 3(d). To extract the information about the area of the fingertip region, adaptive binarization was performed to extract the fingertip region. The fingertip region corresponds to the white regions in Figures 3(b) and 3(d). If the fingertips are toward the camera, only the pixels of the adaptive region of the finger region are extracted with reference to the fingertips (Figure 3(b)). If the fingertips are upward, in other words, if the palm is toward the camera, the entire palm is extracted (Figure 3(d)). The area of the fingertip region was calculated as the number of pixels included in the extracted fingertip region.

\section{GESTURE RECOGNITION}

The process of controlling a smart car menu by using the spatial interaction proposed in the present study is described here. A user may control the smart car menu by using upward, downward, leftward, rightward, and confirmation gestures. The process of each gesture is described here.

Natural directional gestures are used for gesture recognition. The information used for gesture recognition is the coordinate values of the reference point and the area of the hand region. Only the speed of the hand region reference point is used for the recognition of upward, downward, leftward, and rightward gestures. The method of recognizing the leftward and rightward gestures will be described first.

To recognize the leftward and rightward gestures of the hand region reference point, the speed of the reference point coordinates in the $\mathrm{x}$-axis and the $\mathrm{y}$-axis is measured. To reduce the error, the $\mathrm{x}$ and $\mathrm{y}$ coordinate values of the reference points are obtained from three consecutive frames and the average speed is calculated. If the calculated average speed is greater than the speed threshold defined respectively for the left or the right in the $\mathrm{x}$-axis and smaller than the speed threshold defined in the $\mathrm{y}$-axis, the gesture is recognized as a leftward or a rightward gesture.

To recognize the upward and downward gestures of the hand region reference point, the speed of the reference point coordinates in the $\mathrm{x}$-axis and the $\mathrm{y}$-axis is measured. To reduce the error, the $\mathrm{x}$ and $\mathrm{y}$ coordinate values of the reference points are obtained from three consecutive frames and the average speed is calculated. If the calculated average speed is greater than the speed threshold defined respectively for the upside or the downside in the $\mathrm{y}$-axis and smaller than the speed threshold defined in the $\mathrm{x}$-axis, the gesture is recognized as an upward or a downward gesture.

Figure 4 shows a hand gesture to the right side. Figure 4(c) shows the speed plot from the start position of Figure 4(a) to the end position of Figure 4(b). If the speed is greater than the speed threshold defined in Figure 4(c), an event is generated in that direction.

Figure 5 shows a hand gesture to the left side. Figure 5(c) shows the speed plot from the start position of Figure 5(a) to the end position of Figure 5(b). If the speed is greater than the speed threshold defined in Figure 5(c), an event is generated in that direction. 
Figure 6 shows a hand gesture to the upside. Figure 6(c) shows the speed plot from the start position of Figure 6(a) to the end position of Figure 6(b). If the speed is greater than the speed threshold defined in Figure 6(c), an event is generated in that direction.

Figure 7 shows a hand gesture to the downside. Figure 7(c) shows the speed plot from the start position of Figure 7(a) to the end position of Figure 7(b). If the speed is greater than the speed threshold defined in Figure 7(c), an event is generated in that direction.

Figure 8 shows a hand gesture to the front. Figure 8(c) shows the speed plot from the start position of Figure $8(\mathrm{a})$ to the end position of Figure $8(\mathrm{~b})$. If the speed is greater than the speed threshold defined in Figure $8(\mathrm{c})$ and the fingertip area is greater than the threshold, an event is generated in that direction. The information of the fingertip area is used to prevent an erroneous recognition, because upward, downward, leftward, or rightward gestures may be wrongly recognized as a gesture to the front.

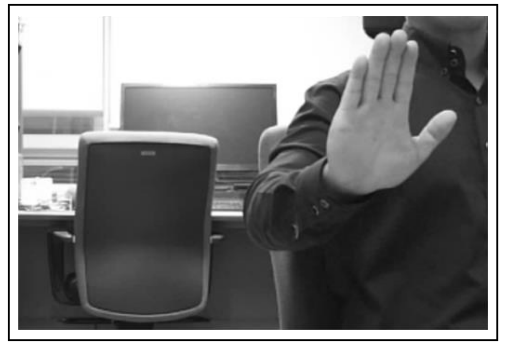

(a)

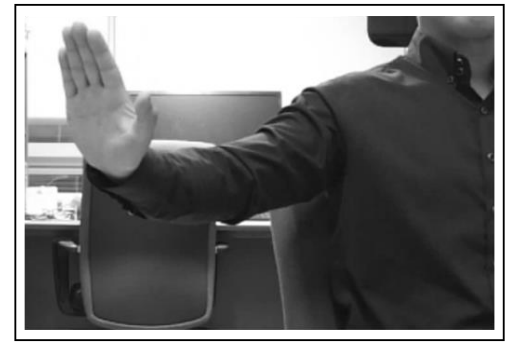

(b)

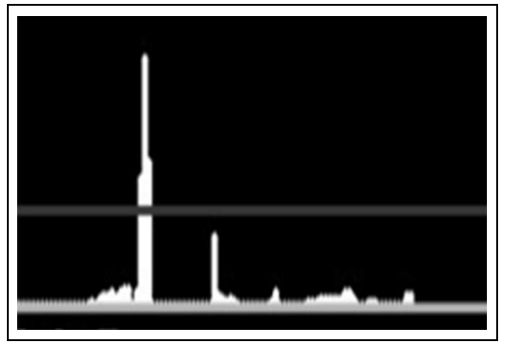

(c)

Figure 4. A rightward gesture

(a) Start position; (b) End position; (c) Speed plot

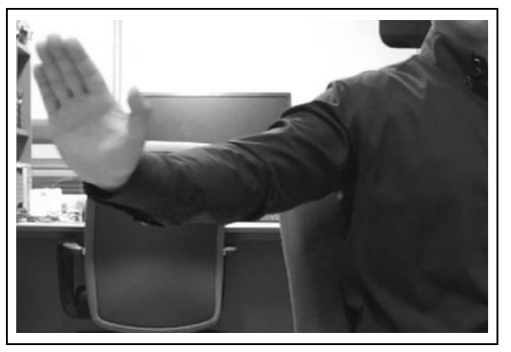

(a)

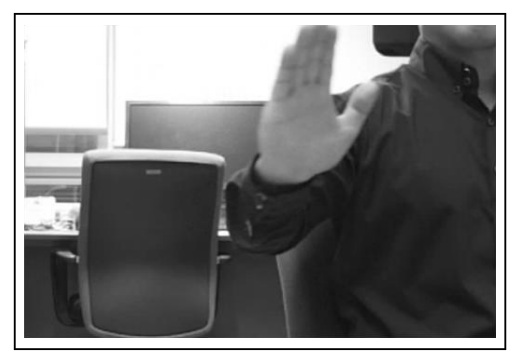

(b)

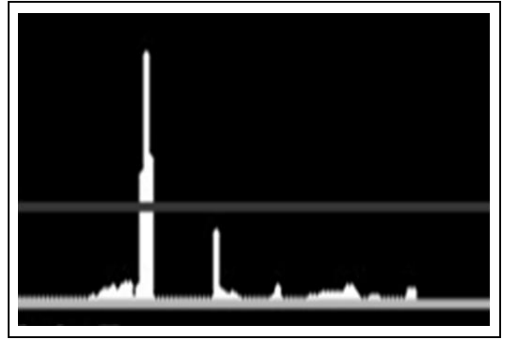

(c)

Figure 5. A leftward gesture

(a) Start position; (b) End position; (c) Speed plot

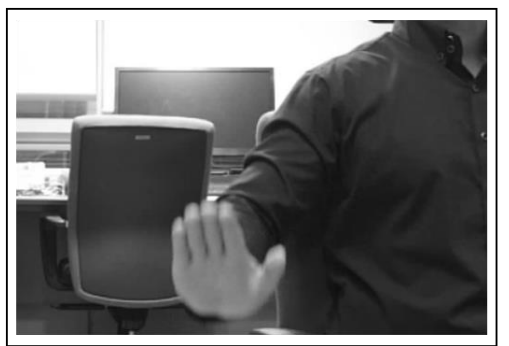

(a)

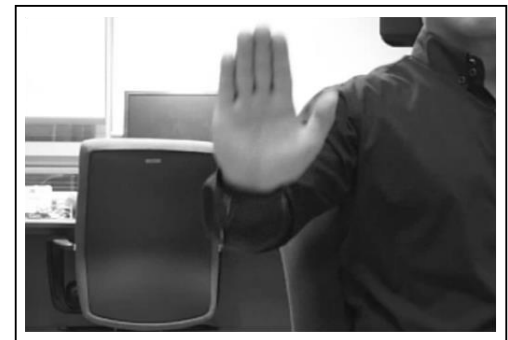

(b)

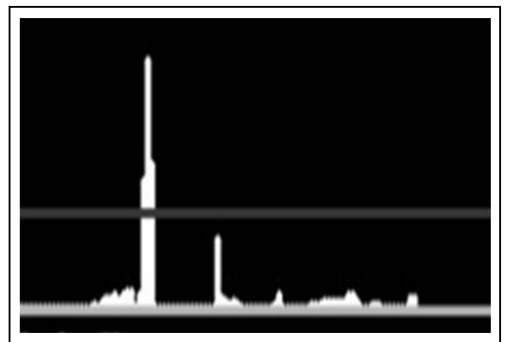

(c)

Figure 6. An upward gesture

(a) Start position; (b) End position; (c) Speed plot 


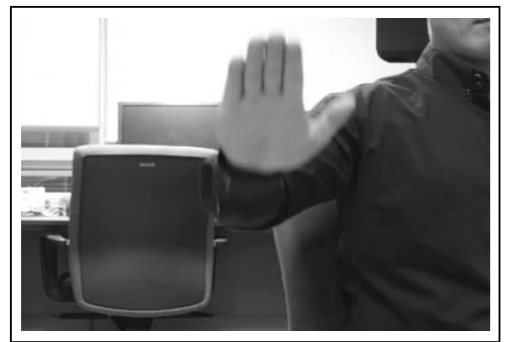

(a)

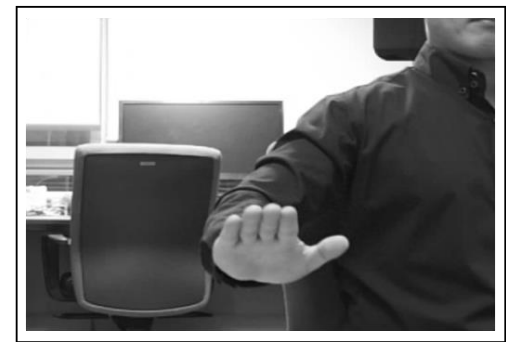

(b)

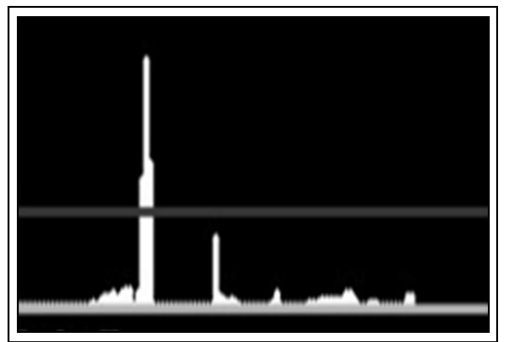

(c)

Figure 7. A downward snap gesture (a) Start position; (b) End position; (c) Speed plot

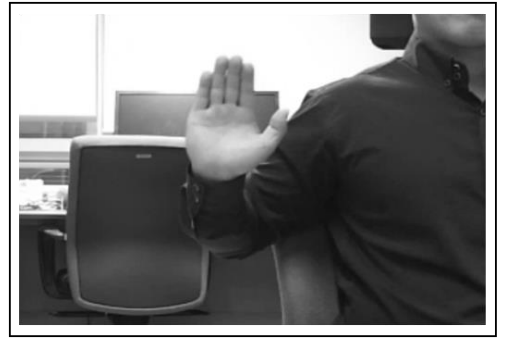

(a)

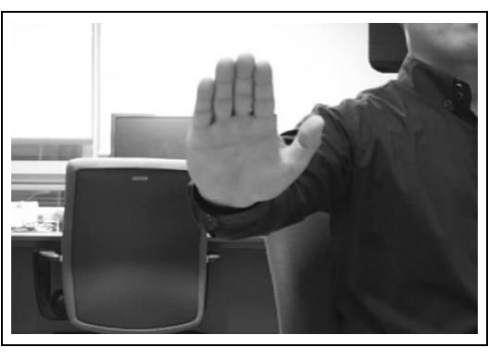

(b)

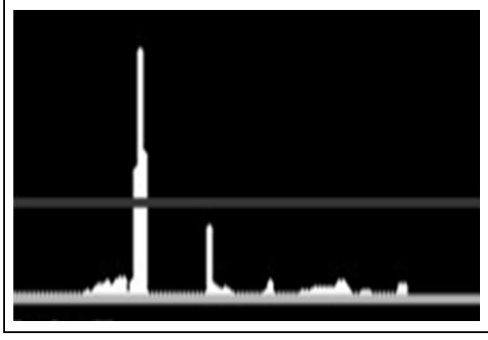

(c)

Figure 8. A gesture to the front

(a) Start position; (b) End positio1n; (c) Speed plot

\section{EXPERIMENTAL RESULTS}

An experiment to test the system was conducted using a personal computer (Intel ${ }^{\circledR}$ Core ${ }^{\mathrm{TM}} \mathrm{i} 7-3770 \mathrm{~K} 3.5$ $\mathrm{GHz} \mathrm{CPU}$ and 8 Gbyte memory) connected to an Intel RealSense depth camera. The rate of gesture recognition was measured to verify the reliability of the system proposed in the present study.

Table 1. Rate of gesture recognition

\begin{tabular}{|c|c|c|c|c|c|c|}
\hline & \multicolumn{5}{|c|}{ Gesture (Number of times) } & \multirow{2}{*}{$\begin{array}{c}\text { Average } \\
(\%)\end{array}$} \\
\cline { 2 - 6 } & Left & Right & Up & Down & Front & 98.2 \\
\hline User 1 & 100 & 100 & 96 & 98 & 97 & 98 \\
\hline User 2 & 99 & 100 & 97 & 98 & 96 & 97.6 \\
\hline User 3 & 98 & 98 & 97 & 100 & 95 & 98.6 \\
\hline User 4 & 100 & 100 & 97 & 98 & 98 & 98.2 \\
\hline User 5 & 97 & 98 & 98 & 99 & 99 & $98.12 \%$ \\
\hline- & - & - & - & - & - & \\
\hline
\end{tabular}

The rate of directional gesture recognition was measured by counting the number of times of recognition of rapid upward, downward, leftward, rightward, and frontward hand gestures. Each of five users made 100 gestures in the experiment to calculate the average rate of recognition. A total of 2453 gestures were successfully recognized out of 2500 gestures, indicating that the rate of gesture recognition was $98.12 \%$ (Table 1). 
Figure 9 shows one user's actual spatial interaction with contents shown on the windshield. Figure 9(a) shows the choice of menu through a frontal gesture made by the user. Figure 9(b) shows the driving according to the customized driving information shown on the display screen.

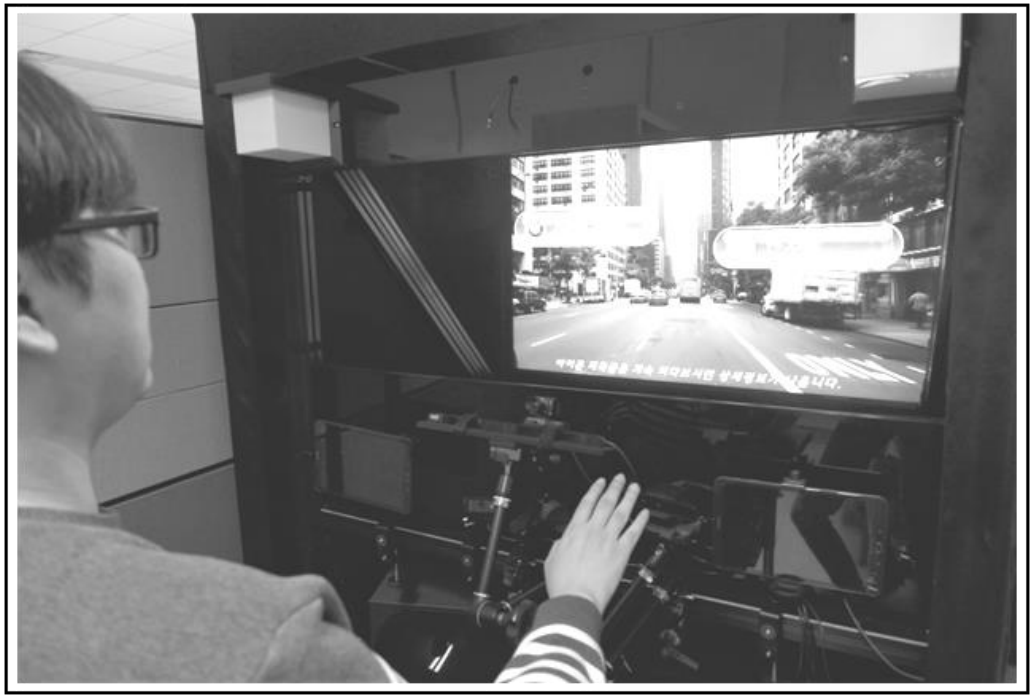

(a)

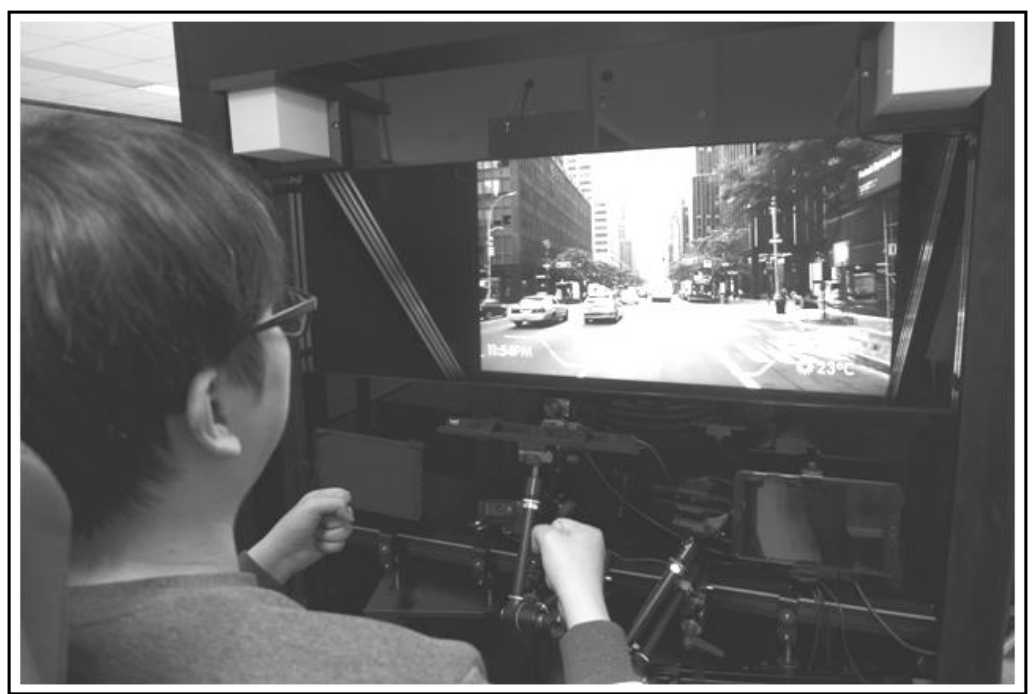

(b)

Figure 9. User test

(a) Menu choice gesture; (b) Car driving

\section{CONCLUSIONS}

This article proposes a method of controlling a screen showing output information on a transparent display of a car windshield using a single depth camera without substantial touches made by user. The configuration of the system for implementing the proposed method was described with the overall flowchart of the system. The system proposed in this article may increase driving safety, because a user may manipulate the screen with only natural gestures while looking at the front, without the need for turning the head to press buttons to control the screen while driving. 


\section{ACKNOWLEDGEMENT}

This research was supported by Korea Creative Content Agency (KOCCA).(No. R2017030009, The development of infotainment contents and interaction for space of movement).

\section{REFERENCES}

Sujin Kwak. et al, 2015. Smart Car Technology and Smart Car-Talk Empirical Environment. Proceeding of Korea Society of Management Information Systems. Republic of Korea, pp 729-738.

Yejin Shin. et al, 2016. Patent Analysis for Innovative Technology Development of Advanced Driver Assistance Systems (ADAS). Journal of Information Technology and Architecture. Vol. 13, No. 1, pp 147-160. 